Case Study

\title{
Rieger Syndrome: A Case with Congenital Absence of Premaxillary Area
}

\author{
Bazopoulou E1*, Karamalikis DD², Kyrkanides S3 , Papadakis E1, Grigoriadou M4 \\ ${ }^{1}$ Department of Oral Pathology and Surgery, School of Dentistry, National and Kapodistrian University of Athens, Greece \\ ${ }^{2}$ Department of Orthodontics, School of Dentistry, National and Kapodistrian University of Athens, Greece \\ ${ }^{3}$ Department of Orthodontics, School of Medicine and Dentistry, Eastman Dental Center, Rochester NY, USA \\ ${ }^{4}$ Department of Genetics, National and Kapodistrian University of Athens, Greece
}

${ }^{*}$ Correspondence author: Euterpe Bazopoulou, Department of Oral Pathology and Surgery, School of Dentistry National and Kapodistrian University of Athens, Greece; Email Id: ebazopou@dent.uoa.gr

Received: September 03, 2018; Accepted: September 10, 2018; Published: September 25, 2018;

\section{Case Study}

Rieger syndrome is characterized by absent maxillary incisor teeth, malformation of the anterior chamber of the eye, and umbilical anomalies [1]. A case with congenital absent of premaxillary area is presented.

The patient, a 10 7/12 -year-old boy, was born to young (father 25, mother 16), non-consanguineous, apparently normal parents, after an uneventful, full-term pregnancy. Hydramnios and a longlasting delivery is reported. He weighed 3000 gr. at birth and had choanal atresia, bilateral aniridia, glaucoma and inverted strabismus. His younger 7-year-old brother and 4-year-old sister are reportedly normal. No similar case among relatives is reported.

Physical and radiologic examination showed absence of premaxllary area and incisor teeth hypodontia and delayed eruption of permanent dentition, short facial height (-3.0 SD) highly arched narrow palate, (narrow free border of soft palate, with small uvula, hypertrophic tonsils), severely short palatal plane (-5.4 $\mathrm{SD})$ and concave skeletal profile $(-5.6 \mathrm{SD})$ posterior displacement of maxillary sinuses and projection of the periumbilical skin (dry palmar skin low posterior hairline). Intelligence was normal.

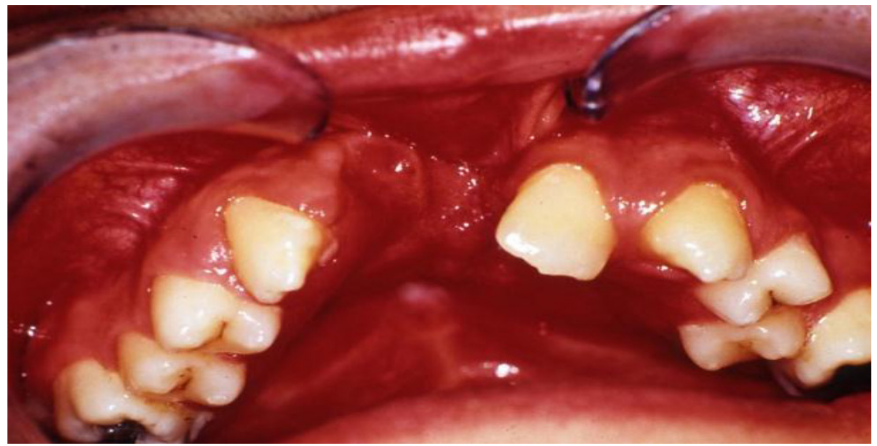

Figure 1. Absence of premaxillary area.

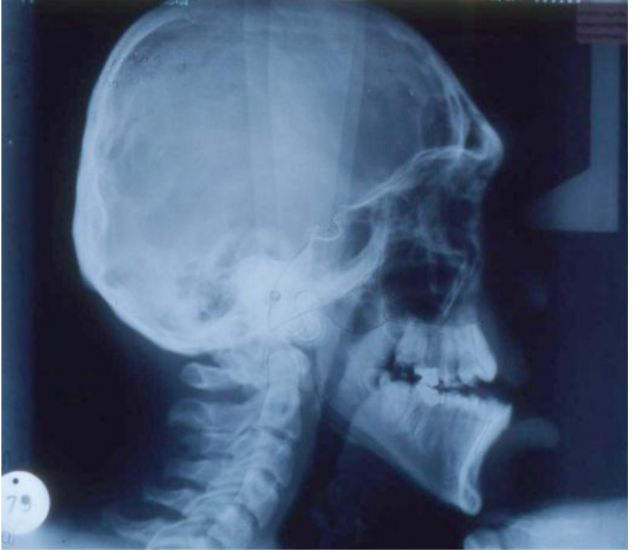

Figure 2. Lateral cephalometric radiography Short Facial Height, concave profile.

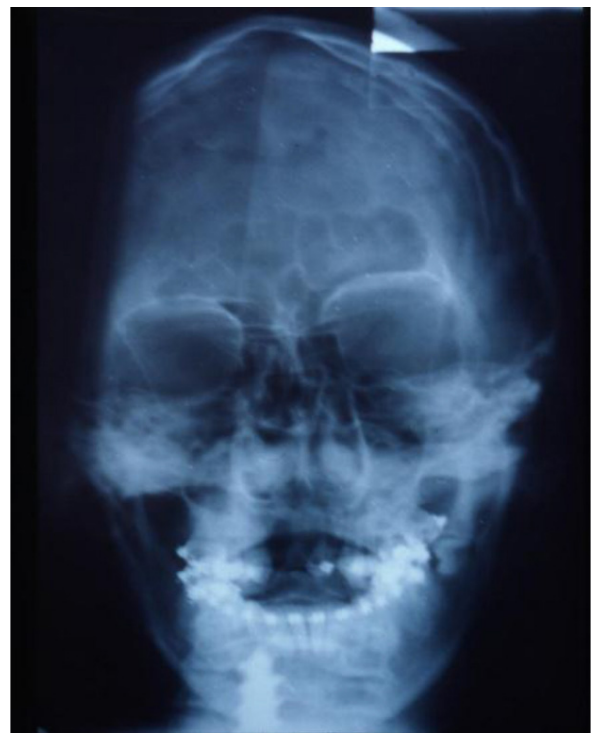

Figure 3. Posterior-front cephalometric radiography. Absence of premaxillary area, infraorbital bony distance. 


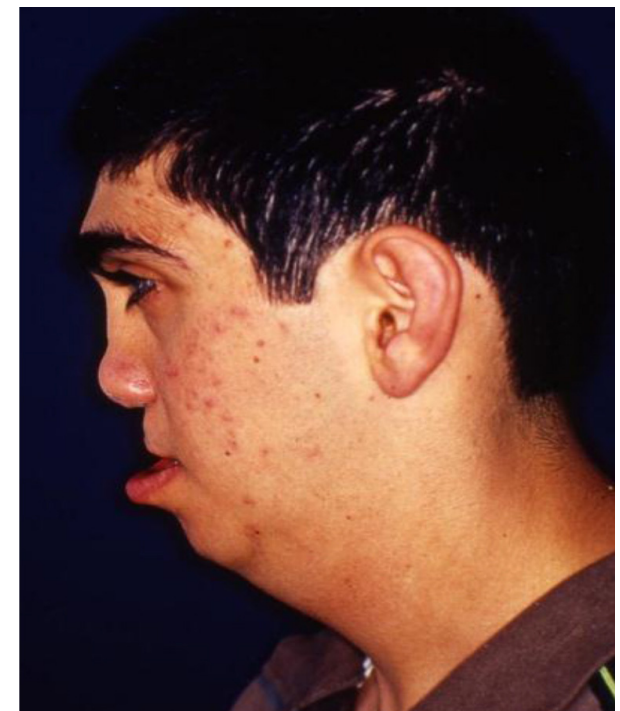

Figure 4. Concave profile.

His karyotype was normal, 46, XY (G-bands).

\section{Panoramic radiograph}

\section{Absent teeth}

$52,51,61,62$

$1813,12,11,21,22,2328$

$4845,43,4131333538$

\section{Cephalometrics}

Patient 10.5 -year-old

Father 35-year-old

\section{Cranial base}

S-N $71.8 \mathrm{~mm}(-2.2 \mathrm{SD})$

$\mathrm{S}-\mathrm{Ba} 44 \mathrm{~mm}(-0.6 \mathrm{SD})$

S-N-Ba $129.6 \mathrm{dg}(0.1 \mathrm{SD})$

SN-FH $151 \mathrm{dg}$ (3.1 SD)

ANS-PNS 43 mm (-5.4 SD)

\section{$70 \mathrm{~mm}-3.5 \mathrm{SD}$ \\ $48 \mathrm{~mm}$ norm \\ $126 \mathrm{dg}$ norm}

$50 \mathrm{~mm}-3.0 \mathrm{SD}$

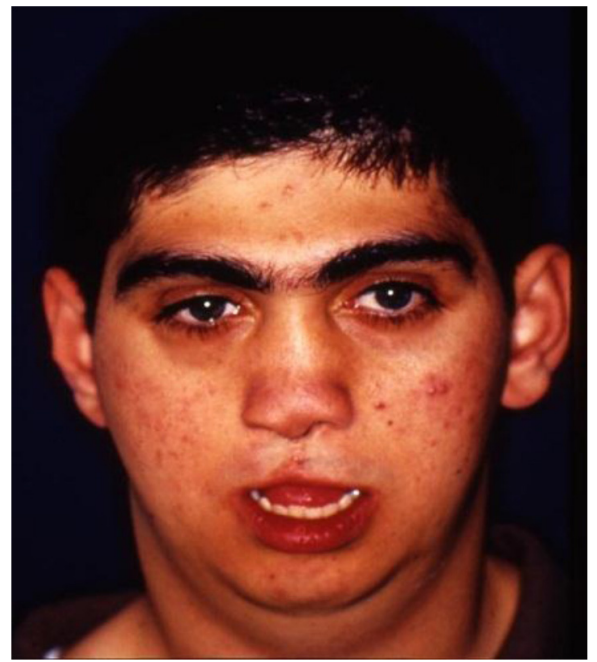

Figure 5. Surgically corrected congenitally absent philtrum.

\section{Skeletal Relations}

Facial Angle $90.6 \mathrm{dg}(2.3 \mathrm{SD}) \quad 88 \mathrm{dg} 3.0 \mathrm{SD}$ (PN-FH)

Lande’s Angle $81.0 \mathrm{dg}(-1.2 \mathrm{SD}) \quad 91 \mathrm{dg} 3.0 \mathrm{SD}$

(AN-FH)

Convexity $-17.8 \mathrm{dg}(-5.6 \mathrm{SD})$

180-(NAP)

$\begin{array}{lll}\text { Vertical Analysis } & & \\ \text { Mandibular Plane } & 20.2 \mathrm{dg}(-1.9) & 23 \mathrm{dg} \\ \text { Y-Axis } & 51.8 \mathrm{dg}(-2.5) & \\ \text { UFH (N-ANS) } & 47.1 \mathrm{~mm}(-2.0) & 61 \mathrm{~mm} \text { norm } \\ \text { TFH (N-Me) } & 106.7 \mathrm{~mm}(-3.0) & 132 \mathrm{~mm} \text { norm } \\ \text { UFH/TFH } & 44.2 \% 43.93 \% & 46.21 \% \text { SNA } 82 \text { dg norm } \\ & & \text { SNB 80 dg norm } \\ & & \text { ANB 2 dg norm }\end{array}$

\begin{tabular}{|l|l|}
\hline $\begin{array}{l}\text { Anterior Cranial Base: } \\
\text { Moderate Short }\end{array}$ & Severely short \\
\hline $\begin{array}{l}\text { Posterior Cranial Base: } \\
\text { Normal }\end{array}$ & normal \\
\hline $\begin{array}{l}\text { Saddle Angle: } \\
\text { Normal }\end{array}$ & normal \\
\hline $\begin{array}{l}\text { Palatal Plane: } \\
\text { Severely short }\end{array}$ & Severely short \\
\hline
\end{tabular}

Maxilla: Mildly retruded to forehead severely protruded to forehead well related to anterior cranial base

Mandible: Prognathic to forehead severely protruded to forehead well related to anterior cranial base

Convexity: Severely decreased; concave skeletal profile Overclosure tendency Maxilla and mandible well related to each other Bony interorbital Distance: $18 \mathrm{~mm} 23 \mathrm{~mm}$

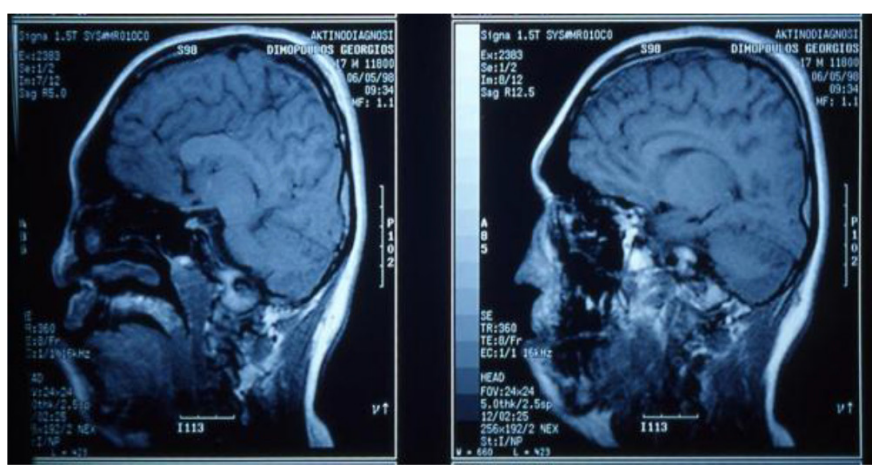

Figure 6. Lateral MRI tomography showing posterior displacement of maxillary sinuses. 


\section{Reference}

1. Gorlin RJ, Cohen Jr, MM Hennekam RCM (2001) Syndromes of the Head and Neck, OXFORD Universal Press. Rieger syndrome (hypodontia and primary mesodermal dysgenesis of the iris). Pp: 1181-1183. 